\title{
Secondary Hairy Cell Leukemia in a Patient With Chronic Myelogenous Leukemia Following Treatment With Tyrosine Kinase Inhibitors: Report of an Extremely Rare Case and Review of the Literature
}

\author{
Reza Setoodeh ${ }^{\mathrm{a}}$, Deniz Peker ${ }^{\mathrm{a}}$, Andrew L. Feldman ${ }^{\mathrm{b}}$, Mark E. Law ${ }^{\mathrm{b}}$, \\ Ling Zhang ${ }^{\mathrm{a}}$, Lynn C. Moscinski ${ }^{\mathrm{a}}$, Haipeng Shao ${ }^{\mathrm{a}, \mathrm{c}}$
}

\begin{abstract}
The occurrence of chronic myelogenous leukemia (CML) and hairy cell leukemia (HCL) in the same patient is extremely rare. The reported cases had either CML and HCL occurring simultaneously or development of CML after HCL. We report an unusual case of CML with subsequent development of HCL after treatment with tyrosine kinase inhibitors. The diagnosis was challenging both clinically and pathologically due to the expected side effects of tyrosine kinase inhibitors and low levels of hairy cells initially. We further showed that the CML and HCL are not clonally related, in contrast to the only case in which clonality study was performed. To our knowledge, this is the first report of HCL developing in patients with CML.
\end{abstract}

Keywords: Secondary hairy cell leukemia; Chronic myelogenous leukemia; Tyrosine kinase inhibitors

\section{Introduction}

The occurrence of chronic myelogenous leukemia (CML)

Manuscript accepted for publication November17, 2011

\footnotetext{
${ }^{a}$ Hematopathology and Laboratory Medicine, H Lee Moffitt Cancer Center and Research Institute, 12902 Magnolia Drive, Tampa, FL, USA

${ }^{\mathrm{b}}$ Department of Laboratory Medicine and Pathology, College Of Medicine, Mayo Clinic, 200 First Street SW, Hilton Building, Room 11-52D, Rochester, MN, USA

${ }^{\mathrm{c}}$ Corresponding author: Haipeng Shao, Hematopathology and Laboratory Medicine, H Lee Moffitt Cancer Center and Research Institute, 12902 Magnolia Drive,Tampa, FL, USA.

Email: Haipeng.Shao@moffitt.org
}

doi:10.4021/jmc404w and hairy cell leukemia (HCL) in the same patient is extremely rare with only 4 cases reported in the literature [1-4]. Two cases showed the coexistence of HCL and CML at the time of diagnosis [1,2], while patients of the other two cases presented with HCL and later developed CML after successful treatment of HCL [3, 4]. Clonal relationship between CML and HCL has been demonstrated in one of the reported cases [2]. The development of HCL after the diagnosis and treatment of CML in our patient is unique and to the best of our knowledge has not been reported before.

\section{Case Report}

A 53-year-old man was diagnosed with chronic myelogenous leukemia chronic phase in October 2006. He presented with splenomegaly and leukocytosis of $50 \times 10^{9} / \mathrm{L}$. The patient was treated with imatinib $400 \mathrm{mg}$ daily and achieved complete remission. After one year of treatment, he started to develop thrombocytopenia and subsequent pancytopenia. The drop in cell counts was attributed to myelosuppression by imatinib therapy. The patient was switched to dasatinib $100 \mathrm{mg}$ daily in November 2008 with no improvement in pancytopenia after multiple dose adjustments. A bone marrow biopsy in January 2009 showed normocellular marrow with erythroid hyperplasia. He was switched to nilotinib in February 2009, and after dose adjustments, his course was still complicated by severe pancytopenia. Two additional bone marrow biopsies in 2010 showed the presence of residual CML by cytogenetic, molecular and fluorescent in situ hybridization studies (FISH) for BCR-ABL1 fusion gene. Cytogenetic and FISH analysis demonstrated $\mathrm{t}(9 ; 22)$ (q34; q11.2) and BCR-ABL1 fusion signals respectively, and RTPCR supported the persistence of residual CML by detecting p210 BCR-ABL1 transcripts. However, the corresponding peripheral blood and bone marrow aspirate smears showed the presence of atypical lymphoid cells with round to bean shaped nuclei, fine chromatin and circumferential cytoplasmic hair-like projections (Fig. 1A). Evaluation of both marrows showed extensive infiltrate by monotonous atypical lymphoid cells with oval nuclei, fine chromatin, and abundant cytoplasm. The infiltrate was associated with marked 

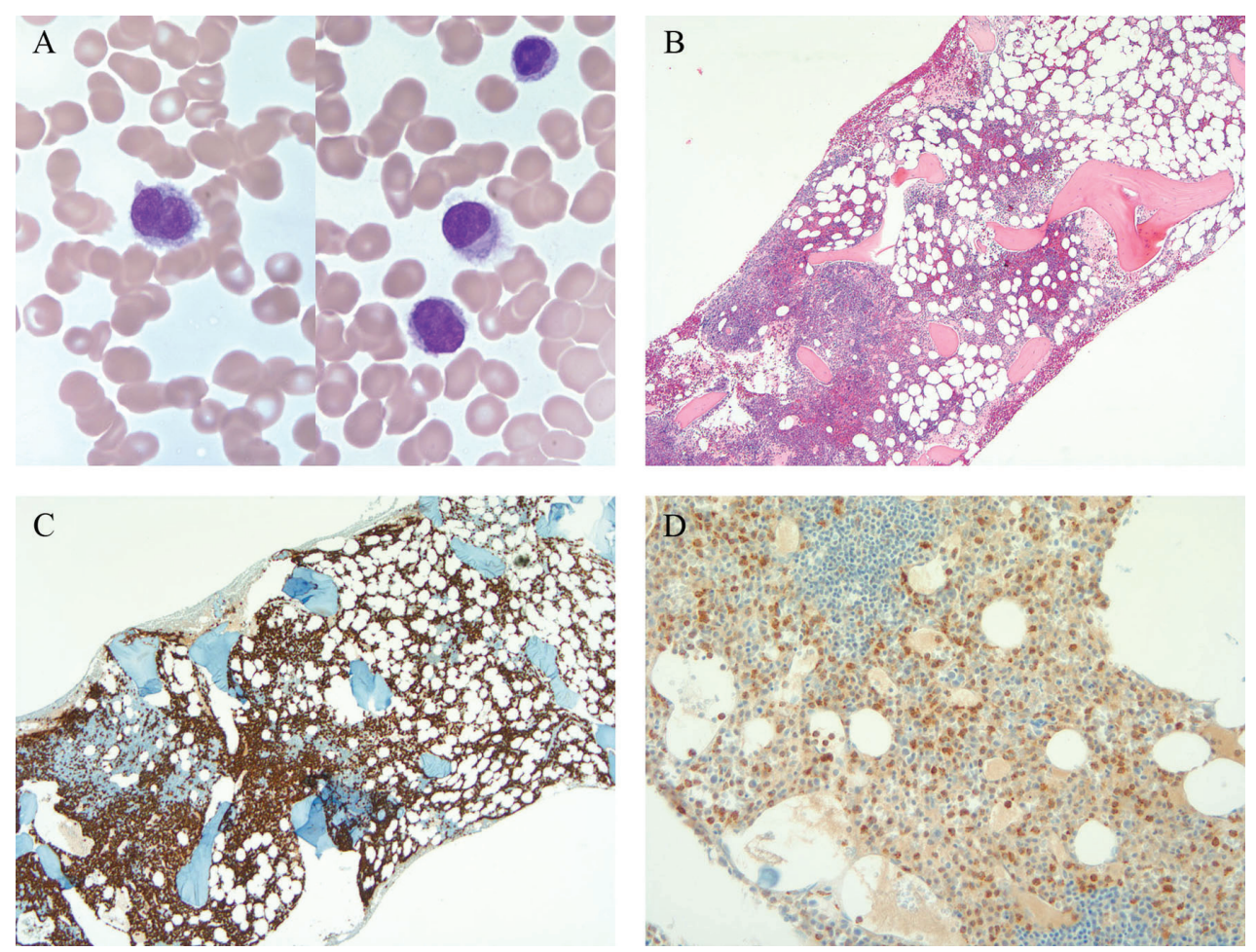

Figure 1. A) Hairy cells in the peripheral blood; B) Bone marrow biopsy showing involvement by atypical lymphoid cells; C) CD20; D) TRAP.

erythroid hyperplasia and myeloid suppression (Fig. 1B). Flow cytometric analysis of the peripheral blood revealed $10 \%$ kappa light chain restricted monoclonal B-cells with expression of CD19, bright CD20, bright CD11c, CD25, and CD103 (data not shown). By flow cytometry, essentially all the B-cells were neoplastic B-cells. Cytochemical stain for
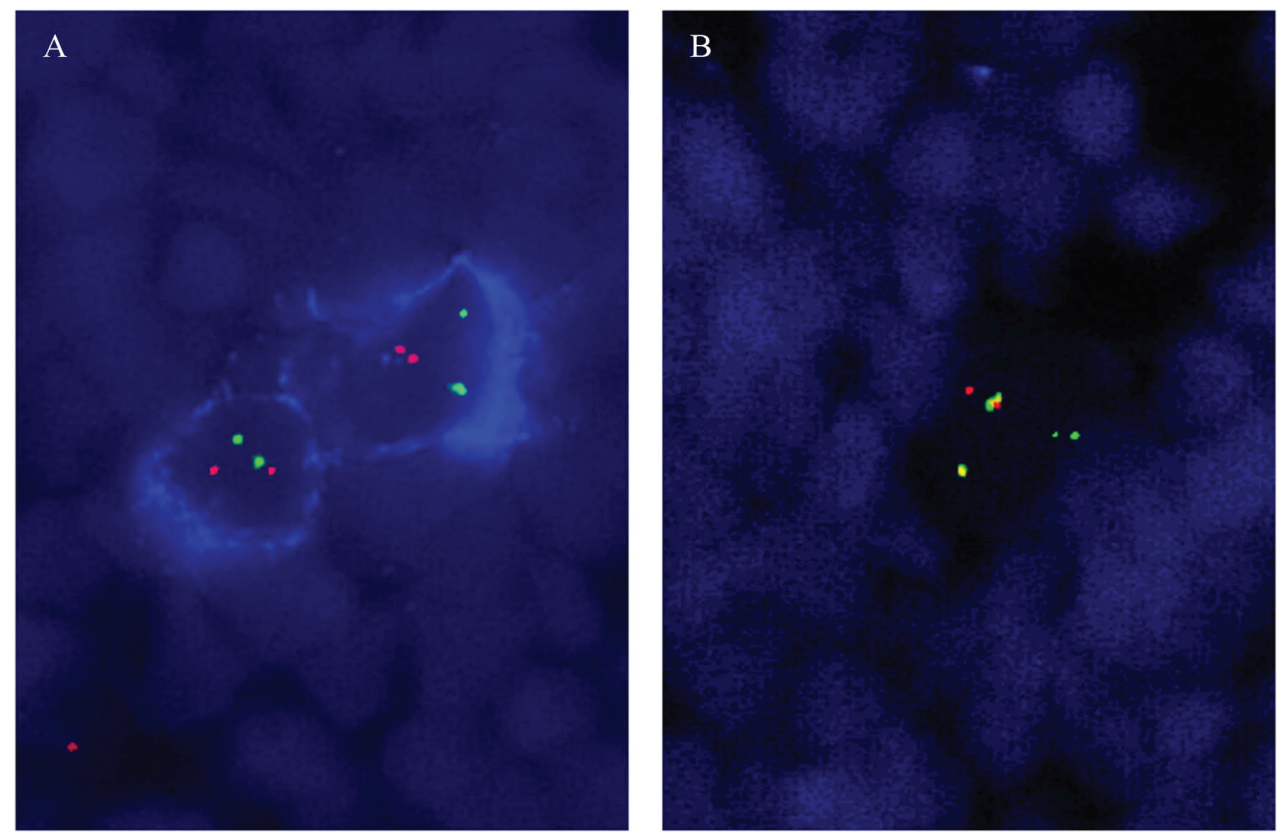

Figure 2. A) FISH demonstrating absence of BCR-ABL1 fusion in the CD20-positive hairy cells; B) Presence of BCR$A B L 1$ fusion in CD20-negative granulocytes. 
tartrate resistant acid phosphatase (TRAP) was strongly positive in a small subset of hairy cells. Immunohistochemical stain for CD20 revealed an extensive infiltrate of B-cells involving approximately $80 \%$ of the marrow (Fig. 1C). A large percentage of the B-cells were strongly positive for TRAP by immunostain (Fig. 1D). Therefore, the morphologic, cytochemical and phenotypic findings were diagnostic of hairy cell leukemia. To determine the clonal relationship between HCL and CML, immuno-FISH was performed on the bone marrow clot sections. The slides were first immunostained with CD20 followed by dual-color, dual-fusion fluorescence in situ hybridization for BCR-ABL1 (Vysis). The immunoFISH demonstrated BCR-ABL1 fusion in the CD20-negative granulocytes (Fig. 2B) but not in the $\mathrm{CD} 20$-positive HCL cells (Fig. 2A), which indicated that the CML and HCL were clonally unrelated.

\section{Discussion}

Chronic myelogenous leukemia (CML) is a clonal myeloproliferative neoplasm caused by constitutive activation of BCR-ABL1 tyrosine kinase produced from the Philadelphia chromosome. Patients with CML typically present with leukocytosis, splenomegaly and anemia. CML is usually effectively treated with tyrosine kinase inhibitors (TKI), which target the BCR-ABL1 tyrosine kinase directly by binding to the kinase domain. The first generation BCR-ABL1 tyrosine kinase inhibitor, imatinib, remains the standard first line therapy for CML and imatinib maintenance therapy is recommended to decrease the risk of relapse [5]. Hairy cell leukemia (HCL) is a rare clonal B-cell lymphoproliferative disorder with distinct clinical and pathological features. Patients with HCL most frequently present with pancytopenia, splenomegaly and circulating hairy cells. HCL cells show a characteristic phenotype with expression of bright CD11c, CD25 and CD103. HCL is typically effectively treated by purine analog therapy with long term remission and normal life span in many patients [6]. The diagnosis of HCL can be difficult, most often due to low levels of circulating HCL cells and subtle bone marrow infiltrate.

In our case the diagnosis of HCL was not suspected in this patient, as myelosuppression with peripheral cytopenia is one of common side effects of tyrosine kinase inhibitors, and both CML and HCL usually present with splenomegaly. Without clinical suspicion, the presence of HCL was neglected in the initial bone marrow biopsy, since the number of circulating HCL cells was low and the bone marrow infiltrate by HCL was inconspicuous. The laboratory work-up for HCL was started only after identification of characteristic hairy cells in the bone marrow aspirates and peripheral blood in repeat biopsies. Therefore, it is always important to keep $\mathrm{HCL}$ in the differential diagnosis of cases with unexplained cytopenia.
Other than developing blastic transformation, patients with CML treated with TKI have no known increased risk of secondary malignancies [7-9]. Most of the secondary tumors in these patients are epithelial cancers and melanoma. The hematologic secondary malignancies include only rare chronic lymphocytic leukemia and diffuse large B-cell lymphoma [9]. Including the HCL reported in this case, all the secondary hematologic malignancies in CML treated with TKI, although rare, are B-cell lymphoproliferative disorders. CML derives from pluripotent stem cells harboring the Philadelphia chromosome, which has been shown to be present in lymphoid cells (B-cells, and less frequently T- cells) other than myeloid cells in CML patients [10]. Therefore, it is conceivable that the pluripotent stem cells may differentiate along the B-cell lineage and develop B-cell lymphoproliferative disorders with additional genomic changes. In fact, in one of the reported cases of coexistent HCL and CML, it has been shown that the HCL and CML cells share the same clonal origin [2]. However, immuno-FISH in this case showed no BCR-ABL1 gene rearrangement in the CD20-positive HCL cells. In contrast, BCR-ABL1 fusion signals were identified in the background granulocytes. Therefore, in this case, the HCL and CML are clonally unrelated independent diseases. It is interesting to note that the patient's CML was successfully treated initially with imatinib with complete remission. The development of HCL coincided with the relapse of CML. It remains an open question of whether CML may facilitate the propagation of the HCL clone.

In summary, we present the first case of HCL that developed as an independent primary neoplasm in CML after TKI therapy. Clinical suspicion for HCL should always be raised in patients with cytopenia, even in the presence of a seemingly plausible explanation, such as side effects of treatments.

\section{Research Funding}

None.

\section{Authors' Disclosures of Potential Conflicts of Interest}

The author(s) indicated no potential conflicts of interest.

\section{References}

1. Orciuolo E, Fazzi R, Galimberti S, Testi C, Azzara A, Carulli G, Petrini M. Chronic myeloid leukaemia and hairy cell leukaemia coexisting in a single patient: difficulties at diagnosis and rational of the therapeutic strategy. Leuk Res. 2006;30(3):349-353. 
2. Pajor L, Kereskai L, Tamaska P, Vass JA, Radvanyi G. Coexistence of chronic myeloid leukemia and hairy cell leukemia of common clonal origin. Cancer Genet Cytogenet. 2002;134(2):114-117.

3. Reeves JE, Robbins BA, Pankey LR, Elias AL, Anderson WF. The simultaneous occurrence of variant hairy cell leukemia and chronic-phase chronic myelogenous leukemia. A case report. Cancer. 1995;75(8):2089-2092.

4. Wandroo FA, Bareford D, el-Jehani F. Chronic myeloid leukaemia occurring in a patient with hairy cell leukaemia. J Clin Pathol. 2000;53(12):940-941.

5. Agrawal M, Garg RJ, Kantarjian H, Cortes J. Chronic myeloid leukemia in the tyrosine kinase inhibitor era: what is the "best" therapy? Curr Oncol Rep. 2010;12(5):302-313.

6. Grever MR. How I treat hairy cell leukemia. Blood. 2010;115(1):21-28.

7. Pilot PR, Sablinska K, Owen S, Hatfield A. Epidemiological analysis of second primary malignancies in more than 9500 patients treated with imatinib. Leukemia. 2006;20(1):148; author reply 149.

8. Roy L, Guilhot J, Martineau G, Larchee R, Guilhot F. Unexpected occurrence of second malignancies in patients treated with interferon followed by imatinib mesylate for chronic myelogenous leukemia. Leukemia. 2005;19(9):1689-1692.

9. Verma D, Kantarjian H, Strom SS, Rios MB, Jabbour E, Quintas-Cardama A, Verstovsek S, et al. Malignancies occurring during therapy with tyrosine kinase inhibitors (TKIs) for chronic myeloid leukemia (CML) and other hematologic malignancies. Blood. 2011;118(16):43534358.

10. Takahashi N, Miura I, Saitoh K, Miura AB. Lineage involvement of stem cells bearing the philadelphia chromosome in chronic myeloid leukemia in the chronic phase as shown by a combination of fluorescence-activated cell sorting and fluorescence in situ hybridization. Blood. 1998;92(12):4758-4763. 